\title{
Enzymatically active single chain caspase-8 maintains T-cell survival during clonal expansion
}

\author{
S Leverrier ${ }^{1}$, GS Salvesen ${ }^{2}$ and CM Walsh ${ }^{\star, 1}$
}

\begin{abstract}
The extrinsic, or death receptor, pathway integrates apoptotic signals through the protease caspase-8 (casp8). Beyond cell death regulation, non-apoptotic functions of casp8 include its essential requirement for hematopoiesis and lymphocyte clonal expansion, and tempering of autophagy in T cells. However, the mechanistic basis for the control of these disparate cellular processes remains elusive. Here, we show that casp8-deficient T-cell survival was rescued by enzymatically active, but not inactive, casp8-expressing retroviruses. The casp8 catalytic induction in proliferating T cell occurred independent of extrinsic and intrinsic apoptotic-signaling cascades and did not induce casp8 proteolytic processing. Using a biotinylated probe selectively targeting enzymatically active caspases, catalytically active full-length casp8 was found in vivo in dividing T cells. A casp8 D387A processing mutant was able to rescue casp8-deficient T-cell proliferation, validating that casp8 self-processing is not required for its non-apoptotic function(s). Finally, casp8 activity was highest in $\mathrm{CDB}^{+} \mathrm{T}$ cells, the most rapidly proliferating subset. These results show that the catalytically competent form of casp8 is required for rapid T-cell proliferation in response to TCR ligation, but that processing of the caspase is only necessary to promote apoptosis.
\end{abstract}

Cell Death and Differentiation (2011) 18, 90-98; doi:10.1038/cdd.2010.69; published online 4 June 2010

On ligation of death receptors (DRs), a complex of proteins essential for inducing apoptosis termed as the death-inducing signaling complex (DISC) is assembled. ${ }^{1}$ Recruited by association with the death domain-containing adaptor FADD, caspase-8 (casp8) is thought to become catalytically active through homodimerization. As an apical caspase, activated casp8 is crucial for most subsequent steps involved in transducing apoptotic signals after DR ligation. Quite unexpectedly, and distinct from the phenotype of micebearing mutations in other apoptotic regulatory factors, a deficiency in casp8 terminates embryonic development at roughly $10.5-12$ days of gestation. ${ }^{2}$ Similar embryonic defects have been observed in mice lacking $\mathrm{FADD}^{3,4}$ or the catalytically inactive casp8 homolog c-FLIP, ${ }^{5}$ suggesting that DISC constituents serve alternate functions in a variety of cell types.

Non-apoptotic functions for casp8, FADD, and c-FLIP have been observed in proliferating lymphocytes. In mice with a T-cell-specific conditional deletion of casp8, T-cell clonal expansion is highly defective. ${ }^{6}$ Similar defects have been observed in mice bearing a transgene encoding a dominantly interfering form of FADD, FADDdd, ${ }^{7-9}$ or in micebearing T-cell-specific conditional deletions of $F A D D^{3,10}$ or C-FLIP. ${ }^{11,12}$ Furthermore, casp8 is essential for human T-cell function, as individuals bearing a single point mutation that renders casp8 non-functional possess few T cells, and the few remaining $\mathrm{T}$ cells are refractory to activation through the $\mathrm{T}$-cell antigen receptor (TCR). ${ }^{13}$ Thus, productive T-cell activation is highly contingent on the function of proteins that are also involved in integrating apoptosis after DR ligation.

Earlier studies have shown that incubation of $T$ cells with the broad range caspase inhibitor zVAD-FMK blocks T-cell proliferation. ${ }^{14,15}$ However, it remains unresolved whether this non-apoptotic function of casp8 requires its catalytic activity. An alternative and plausible scenario is that casp8 may instead serve as a scaffold to orchestrate T-cell signaling. Moreover, if casp8 enzymatic activity is indeed necessary, the mechanistic basis for this non-apoptotic activity in T-cell proliferation and its potential dependency on DR signaling remain elusive. Here, we show using retroviral reconstitution of casp $^{-1-}$ T cells that the catalytic activity of this cysteine protease is essential for T-cell clonal expansion in nonapoptotic cells. We further show that this activity does not depend on extrinsic or intrinsic apoptotic-signaling cascades. Finally, we show that pro-casp8 becomes enzymatically active without autoproteolysis and that such autoprocessing is dispensable for efficient T-cell proliferation. Taken together, these findings show that full-length casp8 catalytic activity is both induced by and necessary for the survival of rapidly dividing T cells, suggesting the existence of differential modes of casp8 activation induced by DR ligation versus mitogen stimulation.

\footnotetext{
${ }^{1}$ Department of Molecular Biology and Biochemistry, Institute for Immunology, University of California, Irvine, CA 92697-3900, USA and ${ }^{2}$ Program in Apoptosis and Cell Death Research, Burnham Institute for Medical Research, La Jolla, CA 92037, USA

${ }^{*}$ Corresponding author: CM Walsh, Department of Molecular Biology and Biochemistry, Institute for Immunology, University of California, Irvine, CA 92697-3900, USA. Tel: + 1949824 8487; Fax: + 1949824 8551; E-mail: cwalsh@uci.edu

Keywords: caspase-8; self-processing; T cells; clonal expansion; apoptosis

Abbreviations: AFC, 7-amino-4-trifluoromethyl coumarin; aomk, acyloxymethyl ketone; casp, caspase; CaspRB, caspase reaction buffer; CFSE, carboxyfluorescein diacetate succinimidyl ester; DED, death effector domain; DISC, death-inducing signaling complex; DR, death receptors; FADD, Fas-associated protein with death domain; FLIP, FLICE-like inhibitory protein; LoadB, loading buffer; pMit, MSCV-IRES-Thy1.1; RIPK1, Rip kinase 1; TCR, T-cell antigen receptor; TNF, tumor necrosis factor; TRAIL, TNF-related apoptosis-inducing ligand; 2DGE, two-dimensional gel electrophoretic

Received 11.6.09; revised 26.4.10; accepted 26.4.10; Edited by JP Medema; published online 04.6.10
} 


\section{Results}

TCR stimulation leads to FADD-dependent induction of IETDase enzymatic activity. To assess caspase catalytic activity in primary $\mathrm{T}$ cells, cell lysates obtained after stimulation with anti-CD3 plus anti-CD28 were incubated with the fluorogenic probes IETD-7-amino-4-trifluoromethyl coumarin (AFC) or DEVD-AFC (optimally recognized by casp8 and casp3, respectively). IETDase activity in T lymphocytes isolated from wild-type (Wt) mice increased over time after stimulation and reached a plateau at roughly $48 \mathrm{~h}$ (Figure 1a). This is consistent with earlier reports ${ }^{14,15}$ showing that chemical caspase inhibitors - although now proven to lack selectivity in complex samples ${ }^{16}$ - block T-cell activation. As a quantitative reference, the IETDase activity observed $36-48 \mathrm{~h}$ after TCR stimulation represents roughly two-thirds of the proteolytic induction measured after DR-induced apoptosis (Supplementary Figure S2B). On activation, FADDdd-expressing $\mathrm{T}$ cells exhibited significantly reduced IETDase activity. As expected, casp8 ${ }^{-1-}$ $\mathrm{T}$ cells possessed greatly diminished IETD-AFC cleavage activity, with the residual activity likely because of other proteases such as casp3 or granzyme B. The decreased activity observed in $F A D D d d \mathrm{~T}$ cells relative to $\mathrm{Wt}$ cannot be attributed to diminished casp8 expression, as casp8 levels were comparable in both genotypes (Figure 1b). Interestingly, although processing of casp8 resulting from cleavage between the large and small subunits of the catalytic domain has been earlier observed in response to DR ligation, immunoblotting analysis revealed that IETDase activity was not accompanied by casp8 processing (see below). IETDase activity in mitogen-stimulated Wt $\mathrm{T}$ cells was not a result of an increased fraction of cells undergoing apoptosis. Indeed, although slightly induced after TCR stimulation, DEVDase activity - one of the most reliable readouts of apoptotic cells - remained comparable among the three different genotypes (Figure 1c). Moreover, the proportion of Annexin-V positive cells remained modestly but consistently higher in FADDdd and casp $^{-1-} \mathrm{T}$ cells (Figure 1d), in accord with earlier studies ${ }^{17,18}$ showing enhanced death in these mutant $T$ cells. Thus, although IETDase activity was greatly enhanced in mitogenically stimulated Wt $\mathrm{T}$ cells, this protease activity, consistent with casp8 activation, did not seem to be linked to apoptotic induction. The enhanced casp8 catalytic activity observed in proliferating $\mathrm{T}$ cells was not selectively induced by CD28 co-receptor stimulation, as similar results were observed after activation with anti-CD3 plus hIL2 (Supplementary Figure S1A). Taken together, these results suggest that an FADD-dependent casp8 activity is induced in non-apoptotic $T$ cells on TCR stimulation, and occurs in the absence of autoproteolytic processing.

As a possible mechanism to eliminate chronically activated lymphocytes, activated T cells induce the expression of death ligands of the TNF family, including FasL, TNF- $\alpha$, and TRAIL. Accordingly, we tested whether TCR-induced casp8 activity was dependent on extrinsic and intrinsic apoptotic-signaling cascades. Inhibition of Fas ligand, TNF- $\alpha$, or TRAIL using Fas-Fc chimeric protein, antagonistic anti-TNF- $\alpha$, and antiTRAIL, respectively, had no impact on IETDase enzymatic
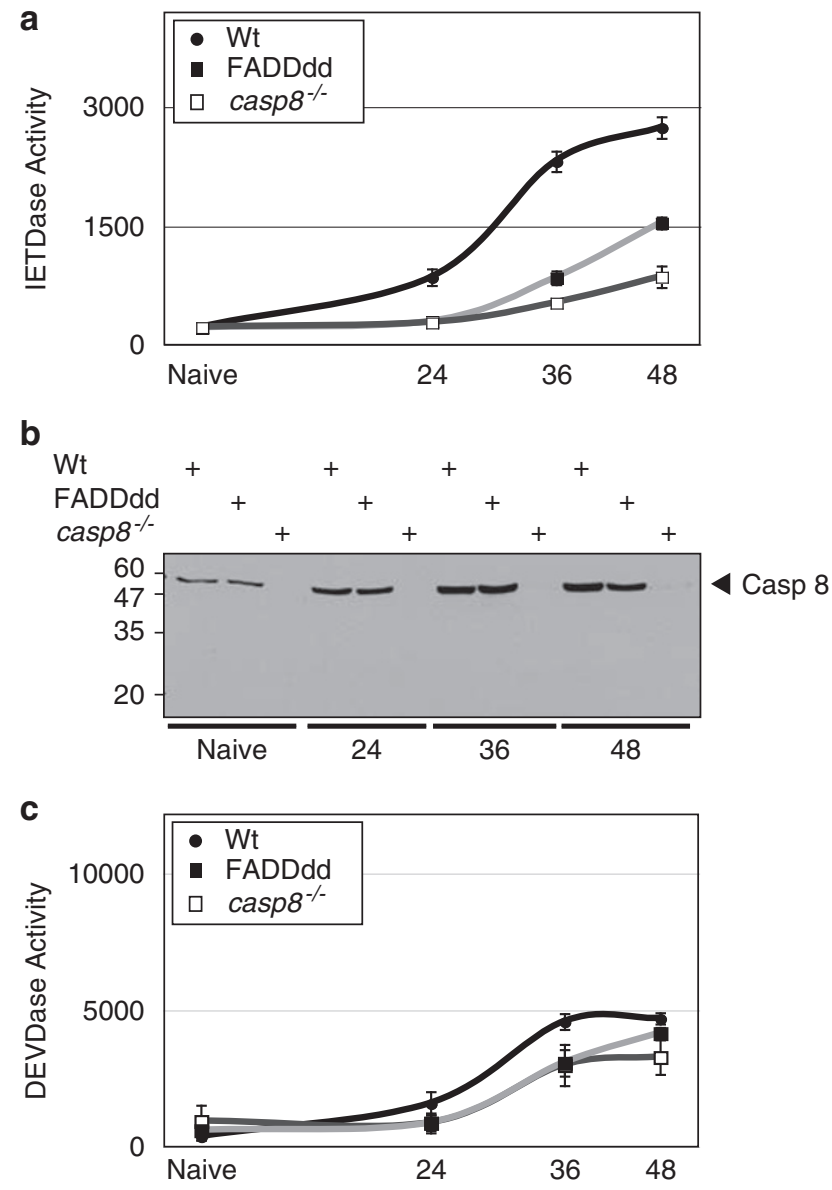

d

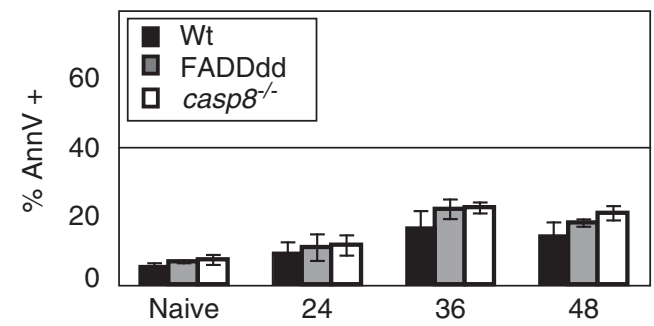

Figure 1 Induction of IETDase activity in non-apoptotic-activated T cells depends on FADD. (a) IETDase induction in mitogenically stimulated $\mathrm{T}$ cells is diminished in FADDdd versus wild-type (Wt) T cells. Wt, FADDdd, or casp8 ${ }^{-/}$ $\mathrm{T}$ cells were activated with plate-bound anti-CD3 $(0.5 \mu \mathrm{g} / \mathrm{ml})$ plus anti-CD28 $(200 \mathrm{ng} / \mathrm{ml})$ for the indicated times. IETDase (a) and DEVDase (c) activity were measured using a synthetic substrate IETD-AFC or DEVD-AFC, respectively, as described in the Materials and methods. (b) Lack of casp8 processing in nonapoptotic-activated T cells. At each time point and for each genotype, a fraction of cells obtained in (a) was processed for casp8 immunoprobing; position of the $55 \mathrm{kDa}$ full-length casp8 is indicated. (d) A fraction of cells was stained with Annexin-V-APC plus 7AAD and the percentages of Annexin- $V$ positive cells were plotted. These experiments were repeated in four mice per genotype and data are expressed as the mean \pm S.E.M.

activity during the course of anti-CD3/CD28 stimulation (Supplementary Figure S1B). Thus, the induction of casp8 activity after TCR stimulation was not due to paracrine or autocrine DR stimulation leading to DISC formation. ${ }^{1}$ The pro-apoptotic protein Bim and the pro-survival factor $\mathrm{Bcl}_{\mathrm{XL}}$, 
both involved in regulating intrinsic apoptosis, are similarly regulated by TCR ligands. To assess the potential regulation of casp8 activity by intrinsic apoptosis regulators, we evaluated casp8 activity in $\mathrm{Bim}^{-1-19}$ or $\mathrm{Bcl}_{\mathrm{XL}}$-overexpressing $^{20} \mathrm{~T}$ cells (Supplementary Figure S1C). TCR ligation of $T$ cells from both mutant mouse lines led to an induction of IETDase activity indistinguishable from Wt T cells. The above data suggest that in non-apoptotic $\mathrm{T}$ lymphocytes, mitogeninduced casp8 activity is independent of extrinsic and intrinsic apoptotic-signaling cascades.

Casp8 catalytic activity confers a survival advantage to proliferating $\mathbf{T}$ cells. Although $\operatorname{casp}^{-1-} \mathrm{T}$ cells remain sensitive to mitogenic stimulation, they fail to proliferate and survive, ${ }^{18}$ suggesting that in this context, casp8 function is to maintain efficient expansion and prevent death. Nevertheless, the importance of casp8 proteolytic activity for T-cell proliferation has not been fully resolved. It is possible that casp8 is essential for T-cell clonal expansion because of a non-enzymatic adaptor function, as casp8 binds to a number of important signaling molecules, including FADD, Rip kinase 1 (RIPK1), and c-FLIP. ${ }^{21}$

To determine whether casp8 catalytic activity is essential for efficient T-cell proliferation, we reconstituted casp8 catalytic activity in mitogenically activated casp $8^{-1-} \mathrm{T}$ cells. We compared the effect of wild-type casp8 (C8-Wt) to a catalytically inactive mutant generated by replacing the essential catalytic cysteine with alanine, casp8-CA (C8-CA). To overcome the inability to transfect naïve murine $T$ cells, casp8-Wt or C8-CA mutant was subcloned into the MSCVIRES-Thy1.1 (pMit) ${ }^{22}$ retroviral vector, which in our experience leads to expression at roughly physiological levels in primary $\mathrm{T}$ cells (see below), a potential concern as overexpression of casp8 can promote apoptosis. ${ }^{23,24}$ Retroviral supernatants of comparable titers were used to infect casp $8^{-1-} \mathrm{T}$ cells mitogenically activated for $24 \mathrm{~h}$. The fold change of Thy 1.1 expression, a marker of T cells infected with the retrovirus, was compared with the initial measurement at d3 (Figure 2a). Thy1.1 expression significantly increased in casp8-Wt-transduced cultures over the 7 days, suggesting that cells reconstituted with Wt catalytically active casp8 possessed a proliferative advantage over control-transduced cells. In contrast, casp8 ${ }^{-1-}$ T cells infected with C8-CA failed to gain a proliferative advantage, supporting the hypothesis that casp8 catalytic activity is crucial for T-cell accumulation after mitogen stimulation.

To determine whether this effect was due to an increased cycling rate or to a survival advantage, we analyzed division of Thy $1.1^{+}$cells using CFSE (Figure 2b). Casp8 ${ }^{-1-} \mathrm{T}$ cells transduced with pMit, casp8-Wt, or C8-CA proceeded through division at roughly equivalent rates, although with very different accumulation profiles, consistent with the notion that casp8 catalytic activity confers a survival advantage. Cells expressing catalytically inactive mutant C8-CA or empty vector initially accumulated, but failed to continue expansion. Strikingly, casp8-Wt-infected cells continued to accumulate over the course of the assay. These findings lead to the interesting conclusion that casp8 catalytic activity does not accelerate cell cycle, but rather maintains the survival of proliferating $\mathrm{T}$ cells.

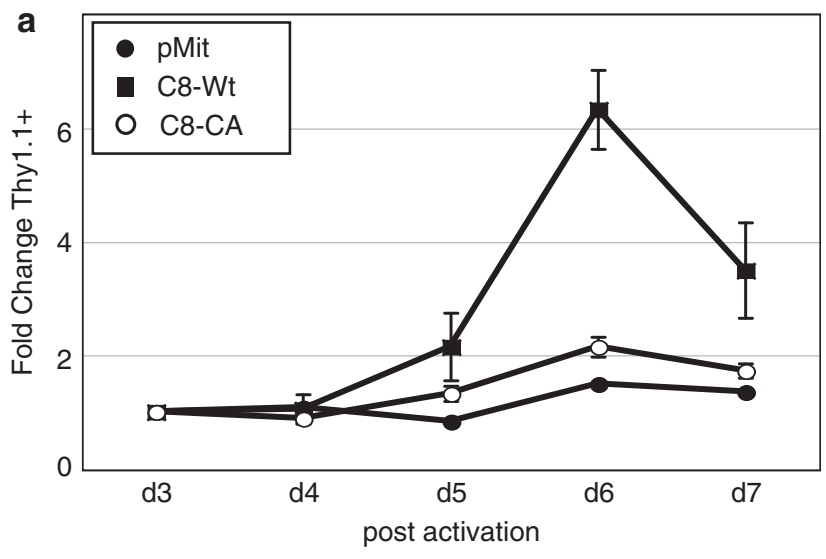

b

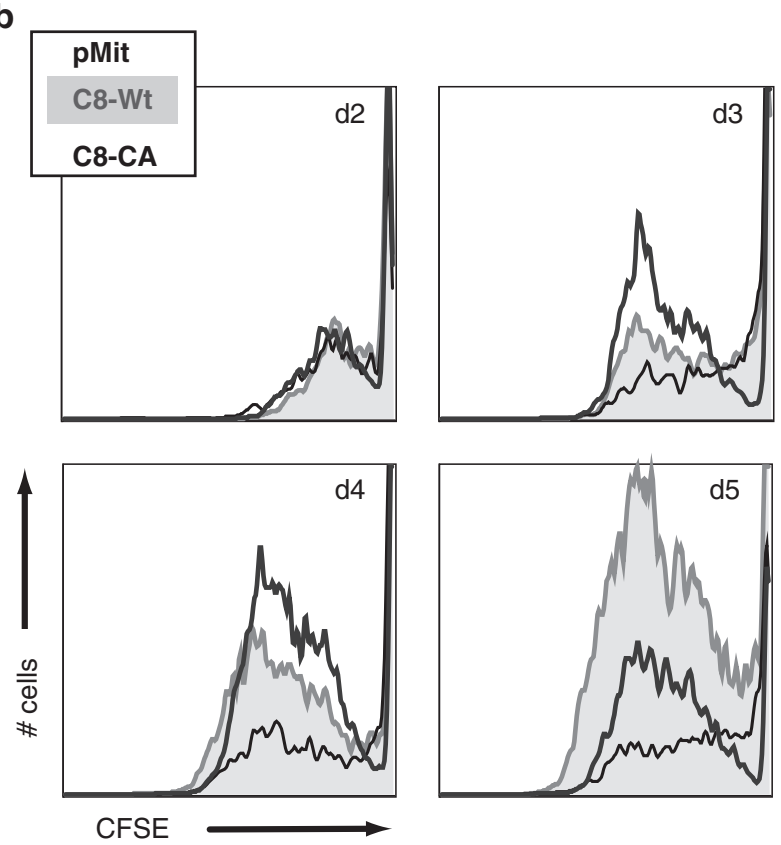

Figure 2 Reconstitution of casp8 catalytic activity in casp $8^{-/-} \mathrm{T}$ cells restores proliferation. (a) Rescue of casp8 $8^{-1} \mathrm{~T}$ cells with catalytically active, but not catalytically inactive casp8. After stimulation with anti-CD3 $(0.5 \mu \mathrm{g} / \mathrm{ml})$ plus antiCD28 $(200 \mathrm{ng} / \mathrm{ml})$ for $24 \mathrm{~h}$, primary casp $8^{-1-}$ T cells were transduced with retroviral supernatants (sups) containing casp8 wild type (C8-Wt) or a catalytically inactive mutant of casp8, C8-CA. Expression of these constructs, as well as the empty vector pMit, was monitored by assessing Thy 1.1 expression by flow cytometry. The fold change in Thy 1.1 expression from 3 to 7 days relative to day 3 was plotted. Data represent the mean \pm S.E.M. of three independent experiments with each vector. (b) Catalytically active casp8 rescues the defective survival of activated casp $8^{-1-}$ T cells. Cycling rate and accumulation profiles of Thy $1.1^{+}$cells, expressing pMit, C8-Wt, or C8-CA, were analyzed by CFSE staining. Representative flow cytometry traces of CFSE dilution histograms are shown; samples were collected for equivalent times

Full-length casp8 is catalytically active in proliferating T cells. Casp8 activation through the DISC has been associated with concomitant autoproteolysis. ${ }^{23-25}$ Such proteolysis, which occurs between the large and small subunits of the catalytic domain, is not required for generation of catalytic activity, but stabilizes the activated enzyme, at least in vitro. ${ }^{25-27}$ Although we found casp8 catalytic activity to be 
essential to the survival of proliferating $T$ cells, we failed to observe any processing of casp8 in TCR-stimulated primary $T$ cells (Figure $1 \mathrm{~b}$ ). Moreover, earlier studies ${ }^{14,15}$ have reported the presence of casp8 proteolytic fragments in activated human $T$ cells, whereas others ${ }^{28}$ have suggested that full-length casp8 is required after TCR stimulation for optimal T-cell activation. We, therefore, hypothesized that the absence of processing may prevent apoptosis and may indicate a distinct mode of casp8 activation. However, the immunoblotting approach used in Figure $1 \mathrm{~b}$ may simply have failed to accurately report casp8 processing during T-cell proliferation.

Even though caspases have been the focus of intense studies, there are few reliable chemical tools available to study specific activity in vivo. ${ }^{16,29}$ The widely used probe, bVAD-fmk, not only produces robust labeling of recombinant caspases, and executioner caspases-3, -6, and -7 in whole cells, but also yields very high background labeling in vivo ( ${ }^{29}$ and data not shown). We thus used an alternative strategy to specifically determine whether the initiator casp8 may become catalytically active without autoproteolytic processing in primary $\mathrm{T}$ lymphocytes. This approach makes use of biotinEVD-acyloxymethyl ketone (bEVD-aomk), a cell-permeant biotinylated activity-based probe that selectively and covalently labels active caspases, ${ }^{30}$ coupled with two-dimensional gel electrophoretic (2DGE) separation to provide a 'fingerprint' of enzymatically active caspase isoforms present in viable cells before lysis. Purified T cells were mitogenically stimulated for $36 \mathrm{~h}$, and during the final hour of culture, bEVD-aomk was added, followed by lysis. As a control for casp8 activation by DR ligation, parallel cultures activated for $24 \mathrm{~h}$ were incubated with anti-Fas for an additional $6 \mathrm{~h}$ before bEVDaomk labeling and cell lysis. When tested for in vivo labeling by bEVD-aomk, full-length endogenous pro-casp8 was found to be linked to the biotinylated substrate in mitogenically activated primary $\mathrm{Wt} T$ cells, as detected by Avidin:BiotinHRP probing of 2DGE blots (Figure 3a), but not in Wt T cells treated with anti-Fas (Figure $3 b$ ). As a control, naive and activated casp $8^{-1-}$ T cells were also submitted to an in vivo labeling by bEVD-aomk, and as expected, neither inactive casp8 nor active casp8 could be detected (Supplementary Figure S2). To analyze the effect of bEVD irreversible binding to casp8 on the protein's pl, we have labeled recombinant pre-activated human casp8 (mutant form lacking the 2 DED domains, $\triangle D E D$-casp8) with bEVD, or with its vehicle DMSO as a control (Supplementary Figure S3).

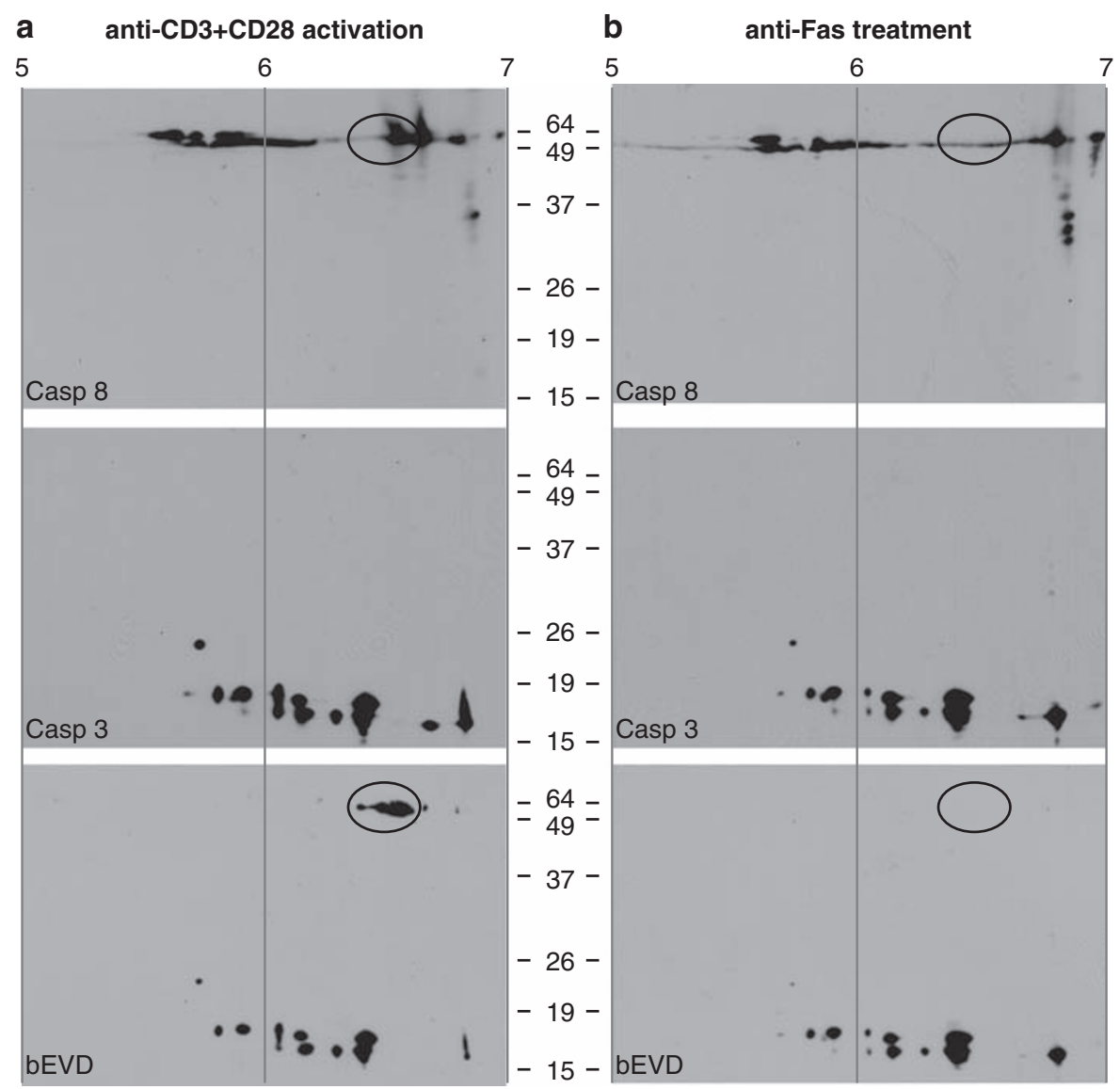

Figure 3 Full-length casp8 is enzymatically active in proliferating T cells. (a) Casp8 fingerprinting assay reveals active pro-casp8 in mitogen-stimulated T cells. Activated caspase labeling was performed in vivo before lysis using a biotinylated probe, $b E V D$, with purified mouse primary T cell following (a) activation for $36 \mathrm{~h}$ with anti-CD3 plus anti-CD28, or (b) incubation of activated T cells with plate-bound anti-Fas $(5 \mu \mathrm{g} / \mathrm{ml})$ for an additional $6 \mathrm{~h}$. Lysates were separated using 2DGE; first dimension based on $\mathrm{pl}$ ( $\mathrm{pH}$ 4-7 range) and second dimension based on molecular weight. Membranes were probed with anti-casp8, anti-casp3, and with an Avidin:Biotinylated-HRP enzyme complex. Immunoblots shown are representative of three independent experiments 
After separation on a 2DGE, both labeled and unlabeled $\triangle \mathrm{DED}$-casp8 lead to several spots between 6.4 and 6.9 , but only the protein incubated with the biotinylated substrate shows an additional spot, suggesting that bEVD binding on casp8 induces a pl shift toward the acidic pole. This shift can be correlated with the appearance of the two active forms (No. 3 and No. 4) in the mitogenically activated sample, suggesting that these two 'more acidic' forms are the result of bEVD binding. As full-length casp8 is not active in anti-Fas-treated cells, only the unlabeled forms (No. 1 and No. 2) are present. These data also suggest that when mitogenically activated $T$ cells are treated with anti-Fas, the pool of catalytically activated full-length casp8 becomes fully processed, most probably by casp6 and/or casp3, providing an amplification loop. This may explain why proliferating $\mathrm{T}$ cells are much more susceptible to DRmediated apoptosis.

Moreover, in these DR-stimulated cells, the overall amount of full-length casp8 was lower than in activated cells, a likely result of casp8 proteolysis. Even though casp8 cleaved products were not detected after $6 \mathrm{~h}$ of anti-Fas treatment - compared with $2 \mathrm{~h}$ post-induction in which $\mathrm{p} 30$ and $\mathrm{p} 18$ were revealed by casp8mAb (Supplementary Figure S4) - we chose this 'relatively late' time point of $6 \mathrm{~h}$ to analyze caspase in vivo activities, as primary naive $T$ cells require a preliminary TCR-mediated activation before being sensitive to anti-Fas, and our objective was to clearly distinguish mitogenically stimulated T cells from apoptotic cells.

Despite modest DEVDase activity in proliferating $T$ cells, casp3 in vivo labeling revealed a series of active forms, corresponding to the p19 and p17 fragments. ${ }^{25}$ It is unlikely that non-specific bEVD-aomk labeling occurred during the lysis procedure, as it was performed under highly denaturing conditions. However, this concern may be significant for IETDase and DEVDase activity assays involving post-lysis incubation with AFC-labeled substrates. A comparison of these experimental procedures revealed further casp3, but not casp8, processing during post-lysis incubation (Supplementary Figure S5A and B). Nevertheless, DEVDase activity remained significantly lower in the non-apoptotic versus antiFas-treated sample (Supplementary Figure S5C). As an additional control, naive $\mathrm{Wt} T$ cells have also been incubated with bEVD-aomk, which failed to reveal active casp8 or casp3 (Supplementary Figure S6). Overall, these results show that full-length endogenous pro-casp8 attains catalytic activity on mitogenic stimulation, but not after DR ligation in T lymphocytes.

Proteolytic processing is not required for casp8 activation in non-apoptotic $\mathbf{T}$ cells. Given the apparent absence of casp8 processing after its catalytic activation in response to mitogenic stimulation of $\mathrm{T}$ cells, we then sought to determine whether blockade of casp8 processing impacts T-cell proliferation. In vitro studies ${ }^{26}$ have shown that a noncleavable mutant of casp8 can dimerize, albeit to a lesser extent than the $\mathrm{Wt}$, and that such dimers retain normal enzymatic activity. ${ }^{27}$ Moreover, a recent study by Wallach and coworkers ${ }^{31}$ has revealed that in $T$ cells expressing a non-cleavable form of casp8, responsiveness to TCR stimulation is not compromised, whereas DR-induced apop- tosis is impacted. To explore this further, we used the aforementioned approach to reconstitute $\operatorname{casp}^{-/-} \mathrm{T}$ cells with a mutant form of the initial self-processing site of casp8 that occurs to separate the two subunits of the catalytic domain (C8-D387A). Activated casp8 ${ }^{-1-}$ T cells were transduced with retroviruses carrying empty vector pMit, the D387A processing mutant or casp8-Wt, and we measured the fold change of Thy $1.1^{+}$retrovirally infected cells. We determined that the non-cleavable casp8-D387A mutant confers a similar survival advantage as casp8-Wt when introduced into casp $8^{-1-} \mathrm{T}$ cells (Figure 4a). Transgeneenforced casp8 expression in casp $8^{-1-} \mathrm{T}$ cells was found to be comparable with that of endogenous casp8 in Wt T cells (Figure 4b). We also observed that the recovery of cycling cells was similar in casp8-Wt and casp8-D387A-expressing cells, and significantly greater than that found for pMittransduced cells (Figure 4c). On TCR stimulation, casp $8^{-/-}$ $\mathrm{T}$ cells reconstituted with either of the above casp8 constructs or with the empty vector exhibited an equivalent level of basal apoptosis, as assessed by the fold change in Annexin-V positive cells (Figure $4 d$ ). However, after a $6 \mathrm{~h}$ incubation with anti-Fas, Annexin- $V$ staining was significantly higher in casp8-Wt-expressing T cells when compared with pMit-, C8-CA-, or casp8-D387A-transduced cells (data not shown). Overall, these results show that casp8 cleavage is dispensable for rescuing the proliferative defect of casp8 $8^{-/}$ $\mathrm{T}$ cells, and suggest that catalytically active full-length casp8 promotes T-cell survival without eliciting an immediate apoptotic response. Further, although casp8 catalytic activity is critical for T-cell survival during proliferation and for DR-induced apoptosis, casp8 processing is only required for the latter.

Differential induction of casp8 enzymatic activity in CD8 versus CD4T cell subsets. Casp $8^{-1-}$ T cells are defective in mitogen-induced proliferation relative to $\mathrm{Wt} \mathrm{T}$ cells, and this defect is particularly acute in $\mathrm{CD}^{+} \mathrm{T}^{+}$lymphocytes, ${ }^{18} \mathrm{a}$ result similarly observed in FADDdd-expressing T cells. ${ }^{22}$ As casp8 catalytic activity promotes survival during T-cell proliferation through a pathway dependent on FADD, we analyzed casp8 activity in both $\mathrm{CD}^{+}{ }^{+}$and $\mathrm{CD}^{+}$subpopulations using magnetic sorting of Wt and FADDdd splenocytes (Figure 5a). After culture with anti-CD3 plus antiCD28, a substantial level of IETDase proteolytic activity was present in $\mathrm{CD}^{+}$cells, with only very modest induction of enzymatic activity in $\mathrm{CD}^{+}$cells. As with unfractionated T cells, a diminished level of IETDase activity was observed in $\mathrm{CD}^{+}$FADDdd cells, reinforcing the link between FADDdependent casp8 induction and T-cell proliferation. To determine whether casp8 catalytic activity in $\mathrm{CD}^{+} \mathrm{T}$ cells was accompanied by efficient proliferation, we reconstituted casp8 catalytic activity in mitogenically activated casp $8^{-1-}$ $\mathrm{T}$ cells - as in Figure $2 \mathrm{a}$ - and compared the effect of C8-Wt in both $\mathrm{CD}^{+}$and $\mathrm{CD}^{+}$subsets (Figure 5b). Thy1.1 expression significantly increased in casp8-Wt $\mathrm{CD}^{+}$transduced cultures over the 7 days, whereas the proliferative advantage gained by $\operatorname{casp}^{-1-} \mathrm{CD} 4^{+} \mathrm{T}$ cells infected with casp8-Wt was significantly lower, showing that casp8 catalytic activity is required to a greater extent in proliferating $\mathrm{CD}^{+}$versus $\mathrm{CD} 4^{+}$T-cell subsets. 
a

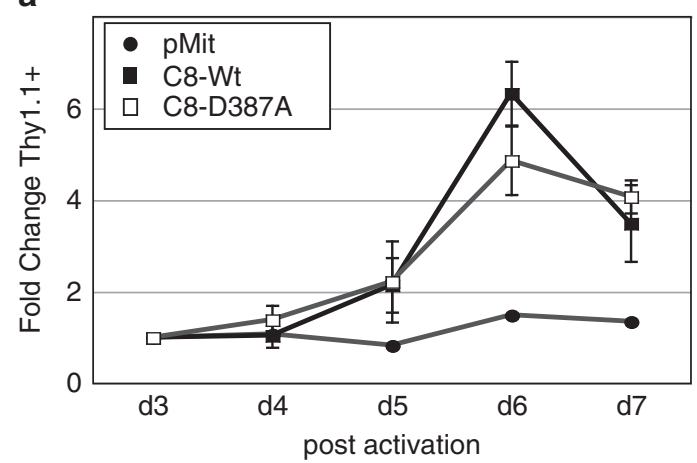

b

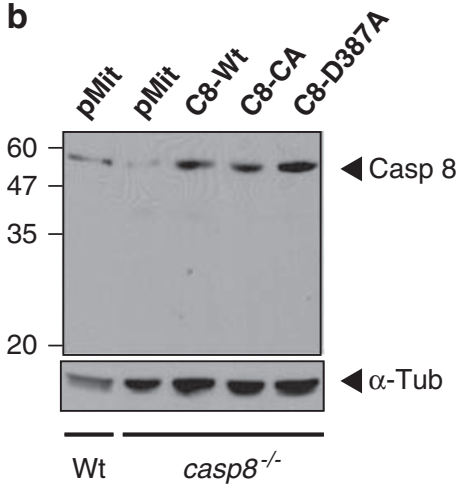

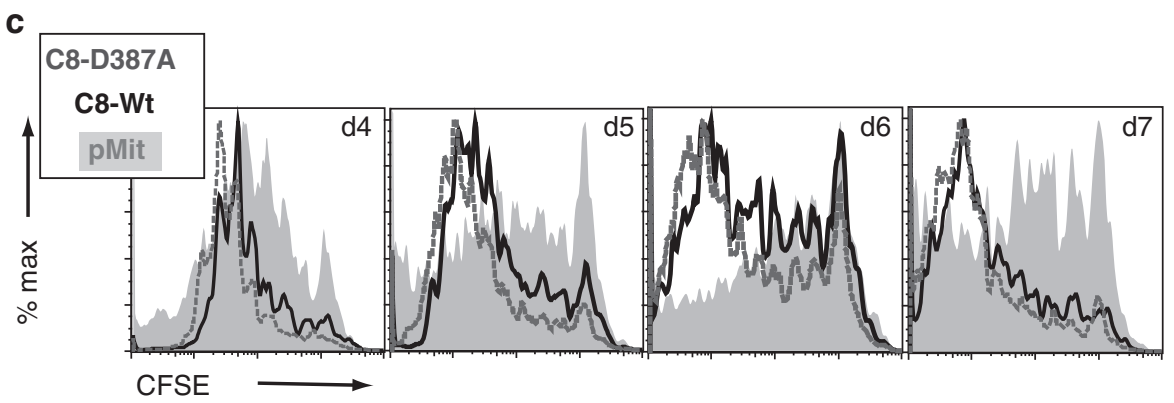

d

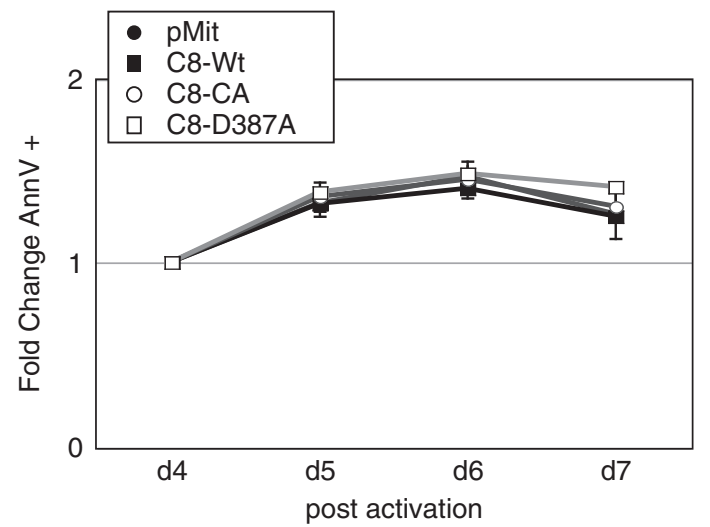

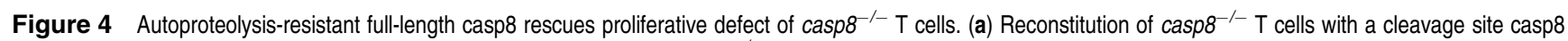
mutant (C8-D387A) restores proliferation. Mitogenically activated primary casp8 ${ }^{-1-}$ T cells were retrovirally transduced with empty vector pMit, casp8 wild type (C8-Wt), or a processing mutant C8-D387A. As in Figure 2, Thy1.1 expression was monitored by flow cytometry and fold change relative to day 3 plotted. Experiment was repeated with three casp8 $8^{-1-}$ mice and results represent the mean \pm S.E.M. (b) Transduction efficiency was assessed by casp8 immunobloting of lysates from T cells expressing the above constructs for 7 days. As a control, wild-type T cells transduced with empty pMit were also loaded. Membrane probed with $\alpha$-tubulin as a loading control. (c) Casp8 processing mutant D387A rescues the defective accumulation of activated casp8 ${ }^{-1-}$ T cells. Cycling rate and accumulation profiles of Thy1.1 $1^{+}$cells, expressing pMit, C8-Wt, or C8-D387A, were evaluated by CFSE staining and cytometry. (d) Percentage of Annexin-V positive cells after transduction of the various casp8 viruses in activated casp8 ${ }^{-/}$ $\mathrm{T}$ cells was monitored by flow cytometry and plotted as fold change relative to day 4 . Mean \pm S.E.M. from three independent experiments plotted for each data point

Such results may trivially be a consequence of differential induction of casp8 expression in $\mathrm{CD}^{+}$versus $\mathrm{CD} 4{ }^{+} \mathrm{T}$ cells. However, levels of pro-casp8 were very similar in both T-cell subsets and in both genotypes, showing that a rise in casp8 expression level is not sufficient to induce its activity, supporting the notion that other regulatory mechanism(s) are involved (Figure 5c). Likewise, differential expression of c-FLIP might account for these findings. Although enhanced by TCR stimulation, c-FLIP expression was similar in CD4 ${ }^{+}$ versus $\mathrm{CD}^{+}{ }^{+} \mathrm{T}$-cell subsets - with only a slight increase in naive Wt $\mathrm{CD}^{+}$cells - discounting the possibility that differential expression of this casp8 regulatory factor accounts for differences in casp8 enzymatic activity between the two subsets. Although c-FLIP is required for T-cell proliferation, ${ }^{11,12}$ changes in its expression in proliferating lymphocytes do not seem sufficient to promote casp8 activity induced by mitogens.

\section{Discussion}

Although caspases are clearly critical mediators in the route to apoptosis, studies from many groups have highlighted a large number of additional cellular functions these cysteine proteases control. ${ }^{32}$ One of these alternative functions is to 

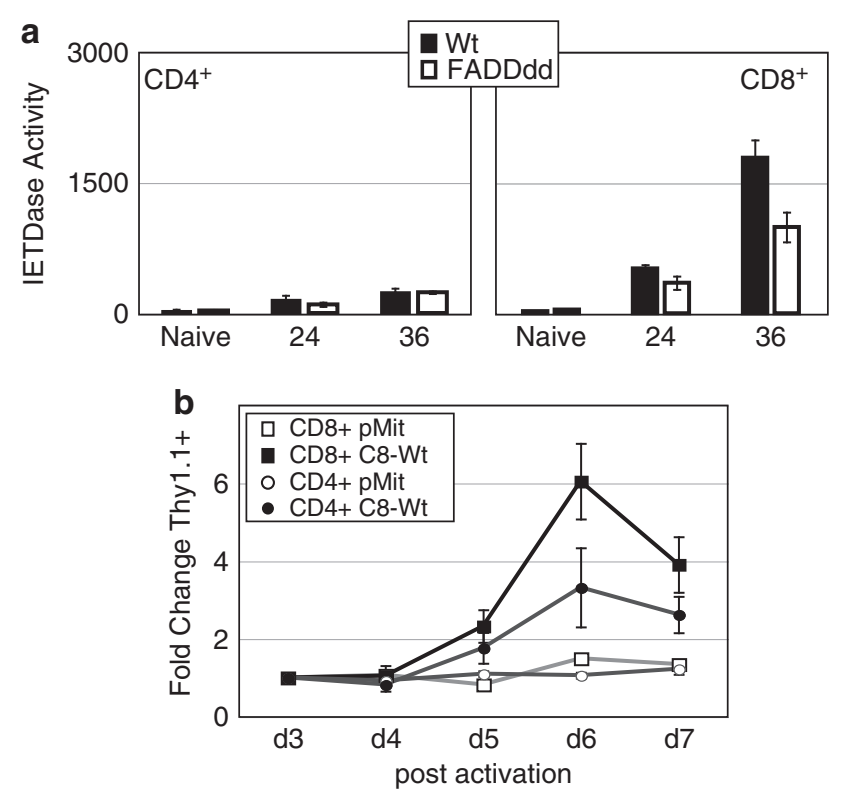

C

C
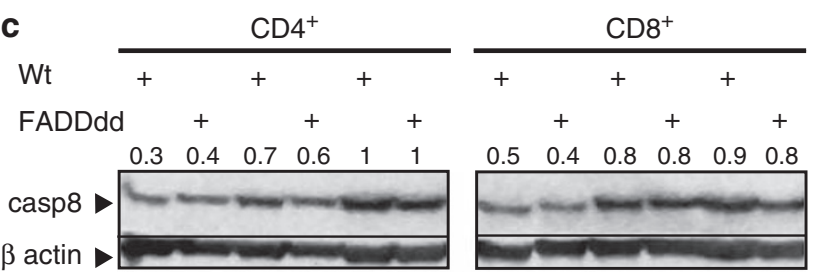

$\begin{array}{llllll}0.5 & 0.4 & 0.8 & 0.8 & 0.9 & 0.8\end{array}$

$\beta$ actin

$\begin{array}{llllll}0.8 & 0.5 & 1 & 0.6 & 0.8 & 0.6\end{array}$
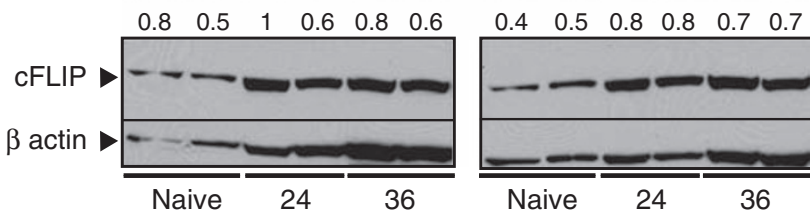

Figure 5 Differential activation of casp8 in mitogenically stimulated CD8 versus CD4 T cells. (a) $\mathrm{CD}^{+}{ }^{+}$, but not $\mathrm{CD} 4^{+}$, T cells exhibit significant IETDase activity after TCR stimulation. $\mathrm{CD}^{+}{ }^{+}$or $\mathrm{CD} 4^{+}{ }^{+}$-cells subsets from Wt and FADDdd mice were magnetically purified using biotin anti-CD8 or biotin anti-CD4, respectively, and then streptavidin microbeads. After stimulation for the indicated times with anti-CD3 plus anti-CD28, IETDase catalytic activity was measured using the fluorogenic synthetic probe IETD-AFC. Experiments replicated using three mice per genotype; bar charts represent the mean \pm S.E.M. (b) Rescue of casp $^{-1-}$ T cells with wildtype casp8 is greater in the $\mathrm{CD}^{+}{ }^{+}$subset. $\mathrm{CD}^{+}$and $\mathrm{CD}^{+}{ }^{+} \mathrm{T}$ cells were stimulated (anti-CD3 plus anti-CD28) and transduced with retroviral supernatantscontaining casp8 wild type (C8-Wt) or the empty vector pMit as in Figure $2 \mathrm{a}$. Expression of these vectors was monitored by assessing Thy 1.1 expression using flow cytometry. The fold change in Thy 1.1 expression from 3 to 7 days relative to day 3 was plotted. Data represent the mean \pm S.E.M. of three independent experiments with each vector and each cellular subset. (c) Similar induction of casp8 and c-FLIP expression in $\mathrm{CD}^{+}{ }^{+}$versus CD8 ${ }^{+} \mathrm{T}$ cells. Western blots of naive or activated $\mathrm{CD} 4^{+}$and $\mathrm{CD} 8^{+} \mathrm{T}$ cells, from either Wt or FADDdd mice, were probed with anti-casp8 or anti-c-FLIP; blots were stripped and reprobed with anti$\beta$-actin as a loading control. Numbers indicate the signal quantification made using ImageJ software. Normalization was performed using $\beta$-actin signal

promote the clonal expansion and survival of mitogenically stimulated lymphocytes. ${ }^{21}$ Here, we have found that nonapoptotic casp8 catalytic activity is induced in cycling CD8 ${ }^{+}$ $\mathrm{T}$ cells. We have further showed that mitogen-dependent casp8 activation is distinct from that induced by DR ligation, with only the latter involving proteolysis in the linker separating the two subunits of the catalytic domain, generally considered to be a result of autoprocessing. However, both processes leading to casp8 activation depend on the activity of FADD. Finally, we have found that C8-Wt and a cleavage-resistant mutant, but not a catalytically inactive mutant form, were capable of rescuing the defective survival of mitogenically stimulated casp $8^{-1-} \mathrm{T}$ cells. These results show that the enzymatic function, but not processing of casp8, is crucial for T lymphocyte clonal expansion.

This work raises several important questions. First, how might casp8 be activated in proliferating lymphocytes independent of DR signaling? A recent report ${ }^{31}$ has shown that T-cell activation is not affected in mice bearing a mutation in the casp8 processing site. Our findings extend that notion by showing that casp8 acquires enzymatic activity in a DR-independent manner without inter-subunit proteolysis, and that this activity maintains T-cell survival and efficient clonal expansion. These findings imply distinct cellular contexts in which casp8 enzymatic activity is facilitated, possibly involving the formation of an analog of the DR-induced DISC. We have recently discovered that casp8 and FADD associate with RIPK1 and the autophagosome-associated proteins Atg5, Atg12, and Atg16L, ${ }^{33}$ suggesting that casp8 is recruited to pre-autophagosomal structures, possibly leading to the induction of its enzymatic activity. FADDdd and casp $8^{-/-}$ T-cells display hyperautophagic morphologies after mitogenic stimulation, and interference with autophagic-signaling rescues these proliferative defects. We have further observed that casp8 activity is dependent on Vps34, an important regulator of pre-autosomal structure development, as its blockade with 3-methyladenine diminished non-apoptotic IETDase induction in cycling Wt T cells. Treatment with the RIPK1-specific inhibitor Necrostatin- $1^{34}$ also completely restored the defective proliferation of $\operatorname{casp} 8^{-/-} \mathrm{T}$ cells, and reduced IETDase activity, suggesting that RIPK1 signaling may be the target of casp8 activity in proliferating $T$ cells. Finally, silencing of RIPK1 in casp $8^{-1-} \mathrm{T}$ cells similarly rescued their proliferation. ${ }^{35}$ Overall, these findings support the concept that during T-cell clonal expansion, casp8 activity is regulated within a DISC-like complex in a DR-independent manner, in which it modulates autophagic signaling. Differential complex assembly may thus explain the distinct modes through which casp8 activity is achieved; DR-induced casp8 activation is accompanied by autoproteolytic cleavage, likely occurring within the DISC, whereas mitogen-induced casp8 activation fails to induce self-processing. This may also explain the selective necessity for FADD and casp8 activity in mitogen- and Ag-stimulated $\mathrm{CD}^{+}{ }^{+} \mathrm{T}_{\text {cells }}{ }^{22,33}$ in which the high rate of cell division observed in these cells may place very stringent energetic demands that necessitate autophagic signaling. However, in this sense, an alternative explanation is simply that $\mathrm{CD}^{+} \mathrm{T}$ cells generate distinct signaling cascades that depend on FADD and casp8.

The means by which active casp8 is restrained from inducing caspase-dependent apoptosis in proliferating lymphocytes also remains an important question, a paradox originally considered within the framework of the initial discovery of non-apoptotic caspase activity in T cells. ${ }^{21}$ One explanation is that the context of casp8 activation itself is important. Our findings indicate that self-processing is 
differentially involved in these seemingly opposing casp8 functions; it is needed for the induction of DR-dependent cell death, but not for triggering T-cell proliferation. The monomeric form of casp8 is activated by dimerization ${ }^{25,27,36}$ and not by proteolysis, and the essential biochemical difference between single chain casp8 and processed (two chain) casp8 seems simply to be a matter of stability of the active dimer. How does this difference play into the distinct biological functions of the protease? Perhaps the higher stability of the cleaved dimer results in a more sustained proteolytic signal. Alternatively, the absence of casp8 proteolysis may limit the potential of this activated protease to interact with downstream targets that would promote apoptosis. Such restriction may conversely enhance substrate selectivity against nonapoptotic targets, such as RIPK1, the kinase domain of which potentiates both autophagic and necroptotic signaling. ${ }^{37,38}$

It is still not clear whether proteolysis in the linker segment correlates with proteolytic removal of the casp8 pro-domain, which contains receptor interacting DEDs. Thus, activated full-length casp8 may also remain associated through its pro-domain to its binding partners and this would entail sequestration of the protease within a restricted subcellular compartment, such as pre-autophagosomal structures. Confinement of enzymatically active casp8 at such structures would prevent free diffusion through the cytosol, potentially limiting its interactions with apoptosis promoting substrates such as casp3 and Bid. This may explain observations described here and elsewhere ${ }^{15}$ of limited casp3 activity in proliferating $\mathrm{T}$ cells as compared with that of apoptotic cells. In addition, other anti-apoptotic proteins may also prevent the elaboration of an apoptotic response in proliferating $\mathrm{T}$ cells until such a response is required to maintain lymphoid homeostasis. Consistent with this, an XIAP deficiency in human $T$ cells leads to apoptotic hypersensitivity in response to both CD95-crosslinking as well as mitogenic stimulation. ${ }^{39}$ Although many important questions remain unanswered, including the identity of activated full-length casp8 interaction partners, substrates, and inhibitors, the study here highlights a vital mechanism for the regulation of casp8 catalytic activity in proliferating versus apoptotic T lymphocytes.

\section{Materials and Methods}

Mice. C57BL/6J Wt mice (Jackson Labs, West Grove, PA, USA), 1017-FADDdd transgenic mice ${ }^{9}\left[\mathrm{Tg}(\mathrm{Lck}-\mathrm{FADD})_{1 \mathrm{Hed}}\right], \mathrm{Bim}^{-1-}$ mice $^{19}$ (Jackson Labs), Bcl $\mathrm{XL}$ transgenic mice (kindly provided by Craig Thompson ${ }^{20}$ ), and casp8 $8^{-1-}$ transgenic mice (FI/FI-CD4-Cre, kindly provided by Stephen Hedrick ${ }^{40}$ ) were bred and maintained in accordance with the institutional animal use and care committee at the UC Irvine vivarium. Age-matched littermates were used as controls.

Antibodies and reagents. Anti-CD3 (2C11.145), anti-CD28, PE anti-Thy1.1, biotin anti-CD4 (L3T4), biotin anti-CD8a (Ly-2), anti-TRAIL (CD253), and anti-TNF- $\alpha$ were from eBioscience (San Diego, CA, USA). Anti- $\beta$-actin and anti- $\alpha$-tubulin were obtained from Abcam (Cambridge, UK). Anti-caspase-8, AC-IETD-AFC, and Ac-DEVD-AFC were supplied by Axxora (San Diego, CA, USA). Anti-caspase-3 was from Cell Signaling (Beverly, MA, USA). Anti-c-FLIP Abs were kindly provided by Dr. J Krowleski. Anti-CD95 (Fas) Jo2 and Fas-Fc chimeric fusion protein were obtained from BD Biosciences (San Jose, CA, USA). Human IL-2 was a kind gift of the Biological Resources Branch, Division of Cancer Treatment and Diagnosis, $\mathrm{NCI}$, Frederick, MD, USA.

T-cell purification and activation. Splenic $\mathrm{T}$ cells were isolated with Easy-Sep Negative Selection kit (Stem Cell Technologies, Vancouver, BC,
Canada). $\mathrm{CD}^{+}$and $\mathrm{CD} 4^{+} \mathrm{T}$ cells were isolated using MACS beads (Miltenyi Biotech, Auburn, CA, USA) according to manufacturer's protocol. Greater than $95 \%$ purity was obtained. Purified T cells were plated at $3 \times 10^{6}$ cells per well containing $0.5 \mu \mathrm{g} / \mathrm{ml}$ plate-bound anti-mouse CD3 and $200 \mathrm{ng} / \mathrm{ml}$ soluble anti-mouse CD28 in $1 \mathrm{ml}$ complete RPMI. ${ }^{17}$ In some cases, co-stimulation was performed with hIL2 $(50 \mathrm{U} / \mathrm{ml})$ instead of anti-CD28. For TNF receptor family inhibition experiments, cells were activated in the presence or absence of Fas-Fc $(2 \mu \mathrm{g} / \mathrm{ml})$, anti-TNF- $\alpha$ $(5 \mu \mathrm{g} / \mathrm{ml})$, and anti-TRAlL $(2 \mu \mathrm{g} / \mathrm{ml})$. At various times after noted stimulation and treatments, cells were harvested, washed, and counted.

IETDase and DEVDase proteolytic activity fluorometric assay. Cell pellets were lysed in 'caspase reaction buffer' (CaspRB) $(100 \mathrm{mM}$ Hepes pH 7.2, $100 \mathrm{mM} \mathrm{NaCl}, 10 \mathrm{mM}$ DTT, $25 \%$ glycerol, $0.1 \%$ CHAPS, $5 \mathrm{mM}$ EDTA, $5 \mu \mathrm{g} / \mathrm{ml}$ leupeptin) for $20 \mathrm{~min}$ on ice. Lysates were cleared at $10000 \times \mathrm{g}$ and a $10^{6}$ cells equivalent volume of lysate was added to $10 \mu \mathrm{M}$ of a synthetic substrate containing an AFC reporter group, Acetyl-IETD-AFC, or Acetyl-DEVD-AFC in a final volume of $100 \mu \mathrm{l}$. After $1 \mathrm{~h}$ incubation (for DEVD-AFC) at room temperature or $3 \mathrm{~h}$ incubation (for IETD-AFC) at $37^{\circ} \mathrm{C}$, activity was read in a 96-well plate fluorometer (Molecular Devices, Sunnyvale, CA, USA) by excitation $405 \mathrm{~nm} / \mathrm{emission} 510 \mathrm{~nm}$, using reaction buffer for background calibration. Substrate cleavage was quantitated using a standard calibration curves established with the free AFC fluorescent molecules using the following formula: relative units of substrate cleavage $=$ fluorescence units/standard curve slope.

Western blot analysis. Lysates were obtained in the same CaspRB as above, or when specified cells were directly lysed in loading buffer (LoadB). Equivalent protein concentrations $(20 \mu \mathrm{g})$ or cell equivalent numbers $\left(10^{6}\right.$ cells) as indicated were loaded on 12.5\% SDS-PAGE (Bradford Assay and pre-cast gels, Bio-Rad, Hercules, CA, USA) and run according to manufacturer's protocol. Proteins were transferred onto PVDF membranes (Millipore, Billerica, MA, USA) for $60 \mathrm{~min}$ at $90 \mathrm{~V}$. Membranes were blocked in TBST containing $5 \%$ milk and incubated overnight at $4{ }^{\circ} \mathrm{C}$ with primary antibody diluted $1: 1000$. Membranes were then washed and incubated with secondary HRP-conjugated antibodies (Vector Labs, Burlingame, CA, USA) diluted $1: 10000$ for $1 \mathrm{~h}$ at RT. Immunoreactive bands were visualized using SuperSignal West Pico or Femto enhanced chemiluminescent reagents (Pierce, Rockford, IL, USA). Quantification and normalization were performed using ImageJ $1.43 \mathrm{r}$ Software from W. Rasband, NIH.

Cell death induction and measurement. A total of $4 \times 10^{6} \mathrm{Wt}$ or casp $8^{-1}$ purified T cells were activated as described above for $24 \mathrm{~h}$, then activated $\mathrm{T}$ cells were cultured onto plate-bound anti-Fas (Jo2) at a final concentration of $5 \mu \mathrm{g} /$ well for the indicated time. In some cases, cells were treated for the last hour of stimulation with bEVD under the same conditions as described below. Cells were then harvested and processed for caspase activity, western blotting, 2DGE analysis, or for Annexin-V staining. For the latter, cells were washed and stained with Annexin-V-APC (Caltag, Bensenville, IL, USA) for $10 \mathrm{~min}$. After washing, cells were resuspended in HBSS containing 7AAD (Invitrogen, Carlsbad, CA, USA) and analyzed on a flow cytometer (FACSCalibur; BD Biosciences, Palo Alto, CA, USA). Percent of Annexin- $\mathrm{V}^{+}$cells was plotted using FlowJo software (Treestar, Ashland, OR, USA)

Retroviral transductions and CFSE analysis. To generate retroviral supernatants, $20 \mu \mathrm{g}$ pMit-vector plus $20 \mu \mathrm{g}$ psi-Eco retroviral packaging vector were co-transfected into 293T using $20 \mathrm{mM} \mathrm{CaCl}{ }_{2}$ and $\mathrm{HBS}$, as described earlier. ${ }^{22}$ Briefly, $5 \mathrm{ml}$ fresh DMEM complete was added $12 \mathrm{~h}$ later and then substituted for $6 \mathrm{ml}$ RPMl complete another $12 \mathrm{~h}$ later. For the next 2 days, every $12 \mathrm{~h}$, media containing virus was collected and replaced by fresh RPMI complete. Virus supernatants were titered in MEFs. For infecting primary $T$ cells, purified $T$ cells were first labeled with $5 \mu \mathrm{M}$ CFDA-SE (Molecular Probes, Eugene, OR, USA) for $8 \mathrm{~min}$ and activated with anti-CD3 $(500 \mathrm{ng} / \mathrm{ml})$ plus anti-CD28 $(200 \mathrm{ng} / \mathrm{ml})$ for $24 \mathrm{~h}$. Cells were then transduced with $1 \mathrm{ml}$ of virus-containing media plus $4 \mu \mathrm{g} / \mathrm{ml}$ polybrene per well and spun at 1800 r.p.m. for $1 \mathrm{~h}$. After $24 \mathrm{~h}$ of infection, cells were washed and re-plated with $50 \mathrm{U} / \mathrm{ml} \mathrm{hlL}-2$. Cells were then harvested, washed, stained with anti-Thy1.1-PE, and anti-CD8-APC for $15 \mathrm{~min}$, and run on FACSCalibur for 2 min per sample. Fold change in Thy 1.1 expression was determined by expressing the ratio of Thy $1.1^{+}$cells at days $3,4,5$, and 7 versus day 3 postactivation in live-gated or $\mathrm{CD}^{+}$-gated populations. CFSE plots are gated on Thy $1.1^{+}$cells. Where noted, a fraction of unlabeled cells was stained with antiThy1.1-PE and processed for Annexin-V binding, as described above. 
In vivo active caspase labeling and 2DGE. Purified T cells were either activated with anti-CD3 plus anti-CD28 for $36 \mathrm{~h}$, or activated in the same condition for $24 \mathrm{~h}$ and then stimulated on plate-bound anti-Fas as described above. In the last hour of culture, the cell permeable biotinylated activity-based probe bEVD-aomk (generous gift from M Bogyo) was added at a final concentration of $10 \mu \mathrm{M}$ to cell media. Cells were then harvested, counted, and equivalent cell numbers $\left(3 \times 10^{6}\right.$ cells) lysed in 2D lysis buffer (8 M Urea, 2\% CHAPS, $50 \mathrm{mM}$ DTT, 0.2\% Bio-Lyte $3 / 10$ ampholytes). As a control, purified naive $T$ cells were incubated under the same conditions with bEVD-aomk and $8 \times 10^{6}$ cells were lysed in 2D buffer. ReadyStrip IPG strips (pH 4-7; Bio-Rad) were rehydrated and loaded with the sample overnight, and isoelectric focusing was performed using a Protean IEF cell, according to manufacturer's instruction (Bio-Rad). Equilibrated strips were then run on a $8-16 \%$ SDS-PAGE at $150 \mathrm{~V}$ (Bio-Lyte 3/10 ampholytes, ReadyStrip IPG strips, Protean IEF cell and pre-cast gels, from Bio-Rad). Proteins were transferred onto PVDF membranes, and anti-casp8 and caspase-3 immunoprobing were carried out as described above. Membranes were stripped with restore western blot stripping buffer (Thermo Scientific, Rockford, IL, USA). The biotinylated substrate was visualized last using a Vectastain $A B C$ kit (Vector Labs), which uses the preformed Avidin:Biotinylated enzyme complex and offers amplification of signal. Reactive spots were revealed using SuperSignal West Pico ( $3 \mathrm{~s}$ exposure for bEVD and $30 \mathrm{~s}$ exposure for casp3), or Femto (10 s for casp8) enhanced chemiluminescent reagents (Pierce). For the naive T-cell sample, SuperSignal West Femto was used and exposure times were increased to $20 \mathrm{~min}$ for casp8, $10 \mathrm{~min}$ for casp3, or $5 \mathrm{~min}$ for bEVD.

\section{Conflict of interest}

The authors declare no conflict of interests.

Acknowledgements. We thank Drs. Stephen Hedrick, Daniel Beisner, and Irene Ch'en for providing the Casp8 ${ }^{F L / F L} \times C D 4$-Cre mice used in these studies. We also thank Dr. Craig Thompson for providing the Bcl-xL transgenic mice, Dr. Matthew Bogyo for the bio-EVD-aomk, and Drs. John Krolewski and Kent Nastiuk for the anti-rat FLIP polyclonal Ab. We appreciate the insightful discussions and critical comments of Drs. David Fruman, Aimee Edinger, Mari Enoksson, and Bryan Bell. This work was supported by NIH grants Al50506 and CA69381.

Authorship Contributions. SL performed experiments and wrote the paper. SL, GS, and CMW designed the research, analyzed data, and wrote the paper.

1. Kischkel F, Hellbardt S, Behrmann I, Germer M, Pawlita M, Krammer P et al. Cytotoxicitydependent APO-1 Fas/CD95 -associated proteins form a death-inducing signaling complex DISC with the receptor. EMBO J 1995; 14: 5579-5588.

2. Varfolomeev EE, Schuchmann M, Luria V, Chiannilkulchai N, Beckmann JS, Mett IL et al. Targeted disruption of the mouse caspase 8 gene ablates cell death induction by the TNF receptors, Fas/Apo1, and DR3 and is lethal prenatally. Immunity 1998; 9: 267-276.

3. Zhang J, Cado D, Chen A, Kabra N, Winoto A. Fas-mediated apoptosis and activationinduced T-cell proliferation are defective in mice lacking FADD/Mort1. Nature 1998; 392: 296-300.

4. Yeh WC, Pompa JL, McCurrach ME, Shu HB, Elia AJ, Shahinian A et al. FADD: essential for embryo development and signaling from some, but not all, inducers of apoptosis. Science 1998; 279: 1954-1958.

5. Yeh WC, Itie A, Elia AJ, Ng M, Shu HB, Wakeham A et al. Requirement for Casper (c-FLIP) in regulation of death receptor-induced apoptosis and embryonic development. Immunity 2000; 12: 633-642.

6. Salmena L, Lemmers B, Hakem A, Matysiak-Zablocki E, Murakami K, Au PY et al. Essential role for caspase 8 in T-cell homeostasis and T-cell-mediated immunity. Genes Dev 2003; 17: 883-895.

7. Newton K, Harris A, Bath M, Smith K, Strasser A. A dominant interfering mutant of FADD/ MORT1 enhances deletion of autoreactive thymocytes and inhibits proliferation of mature T lymphocytes. EMBO J 1998; 17: 706-718.

8. Zornig M, Hueber AO, Evan G. p53-dependent impairment of T-cell proliferation in FADD dominant-negative transgenic mice. Curr Biol 1998; 8: 467-470.

9. Walsh C, Wen B, Chinnaiyan A, O'Rourke K, Dixit V, Hedrick S. A role for FADD in T cell activation and development. Immunity 1998; 8: 439-449.

10. Zhang Y, Rosenberg S, Wang H, Imtiyaz HZ, Hou YJ, Zhang J. Conditional Fas-associated death domain protein (FADD): GFP knockout mice reveal FADD is dispensable in thymic development but essential in peripheral T cell homeostasis. J Immunol 2005; 175: 3033-3044.
11. Chau H, Wong V, Chen NJ, Huang HL, Lin WJ, Mirtsos C et al. Cellular FLICE-inhibitory protein is required for T cell survival and cycling. J Exp Med 2005; 202: 405-413.

12. Zhang N, He YW. An essential role for c-FLIP in the efficient development of mature T lymphocytes. J Exp Med 2005; 202: 395-404.

13. Chun HJ, Zheng L, Ahmad M, Wang J, Speirs CK, Siegel RM et al. Pleiotropic defects in lymphocyte activation caused by caspase-8 mutations lead to human immunodeficiency. Nature 2002; 419: 395-399.

14. Kennedy NJ, Kataoka T, Tschopp J, Budd RC. Caspase activation is required for T cell proliferation. J Exp Med 1999; 190: 1891-1896.

15. Alam A, Cohen LY, Aouad S, Sekaly RP. Early activation of caspases during T lymphocyte stimulation results in selective substrate cleavage in nonapoptotic cells. J Exp Med 1999; 190: $1879-1890$.

16. McStay GP, Salvesen GS, Green DR. Overlapping cleavage motif selectivity of caspases: implications for analysis of apoptotic pathways. Cell Death Differ 2008; 15 : 322-331.

17. Arechiga AF, Bell BD, Solomon JC, Chu IH, Dubois CL, Hall BE et al. Cutting edge: FADD is not required for antigen receptor-mediated NF- $\{$ kappal\}B activation. J Immunol 2005; 175: $7800-7804$.

18. Arechiga AF, Bell BD, Leverrier S, Weist BM, Porter M, Wu Z et al. A Fas-associated death domain protein/caspase-8-signaling axis promotes S-phase entry and maintains $\mathrm{S} 6$ kinase activity in T cells responding to IL-2. J Immunol 2007; 179: 5291-5300.

19. Bouillet P, Metcalf D, Huang DC, Tarlinton DM, Kay TW, Kontgen F et al. Proapoptotic Bcl-2 relative Bim required for certain apoptotic responses, leukocyte homeostasis, and to preclude autoimmunity. Science 1999; 286: 1735-1738.

20. Chao DT, Linette GP, Boise LH, White LS, Thompson CB, Korsmeyer SJ. Bcl-XL and Bcl-2 repress a common pathway of cell death. J Exp Med 1995; 182: 821-828.

21. Siegel RM. Caspases at the crossroads of immune-cell life and death. Nat Rev Immunol 2006; 6: 308-317.

22. Beisner DR, Chu IH, Arechiga AF, Hedrick SM, Walsh CM. The requirements for fasassociated death domain signaling in mature T cell activation and survival. J Immunol 2003; 171: 247-256

23. Muzio M, Chinnaiyan A, Kischkel F, O'Rourke K, Shevchenko A, Ni J et al. FLICE, a novel FADD-homologous ICE/CED-3-like protease, is recruited to the CD95 (Fas/APO-1) death - inducing signaling complex. Cell 1996; 85: 817-827.

24. Boldin M, Goncharov T, Goltsev $\mathrm{Y}$, Wallach D. Involvement of $\mathrm{MACH}$, a nove MORT1/FADD-interacting protease, in Fas/APO-1- and TNF receptor-induced cell death. Cell 1996; 85: 803-815.

25. Boatright KM, Renatus M, Scott FL, Sperandio S, Shin H, Pedersen IM et al. A unified model for apical caspase activation. Mol Cell 2003; 11: 529-541.

26. Donepudi M, Mac Sweeney A, Briand C, Grutter MG. Insights into the regulatory mechanism for caspase-8 activation. Mol Cell 2003; 11: 543-549.

27. Pop C, Fitzgerald P, Green DR, Salvesen GS. Role of proteolysis in caspase-8 activation and stabilization. Biochemistry 2007; 46: 4398-4407.

28. Su H, Bidere N, Zheng L, Cubre A, Sakai K, Dale J et al. Requirement for caspase-8 in NF-kappaB activation by antigen receptor. Science 2005; 307: 1465-1468.

29. Pop C, Salvesen GS, Scott FL. Caspase assays: identifying caspase activity and substrates in vitro and in vivo. Methods Enzymol 2008; 446: 351-367.

30. Kato D, Boatright KM, Berger AB, Nazif T, Blum G, Ryan C et al. Activity-based probes that target diverse cysteine protease families. Nat Chem Biol 2005; 1: 33-38.

31. Kang TB, Oh GS, Scandella E, Bolinger B, Ludewig B, Kovalenko A et al. Mutation of a selfprocessing site in caspase-8 compromises its apoptotic but not its nonapoptotic functions in bacterial artificial chromosome-transgenic mice. J Immunol 2008; 181: 2522-2532.

32. Lamkanfi M, Festiens N, Declercq W, Vanden Berghe T, Vandenabeele P. Caspases in cell survival, proliferation and differentiation. Cell Death Differ 2007; 14: 44-55.

33. Bell BD, Leverrier S, Weist BM, Newton RH, Arechiga AF, Luhrs KA et al. FADD and caspase-8 control the outcome of autophagic signaling in proliferating $T$ cells. Proc Natl Acad Sci USA 2008; 105: 16677-16682.

34. Degterev A, Hitomi J, Germscheid M, Ch'en IL, Korkina O, Teng X et al. Identification of RIP1 kinase as a specific cellular target of necrostatins. Nat Chem Biol 2008; 4: 313-321.

35. Ch'en IL, Beisner DR, Degterev A, Lynch C, Yuan J, Hoffmann A et al. Antigen-mediated T cell expansion regulated by parallel pathways of death. Proc Natl Acad Sci USA 2008; 105: 17463-17468.

36. Keller N, Mares J, Zerbe O, Grutter MG. Structural and biochemical studies on procaspase8: new insights on initiator caspase activation. Structure 2009; 17: 438-448.

37. Degterev A, Huang Z, Boyce M, Li Y, Jagtap P, Mizushima N et al. Chemical inhibitor of nonapoptotic cell death with therapeutic potential for ischemic brain injury. Nat Chem Biol 2005; 1: 112-119.

38. Hitomi J, Christofferson DE, Ng A, Yao J, Degterev A, Xavier RJ et al. Identification of a molecular signaling network that regulates a cellular necrotic cell death pathway. Cell 2008; 135: 1311-1323.

39. Rigaud S, Fondaneche MC, Lambert N, Pasquier B, Mateo V, Soulas P et al. XIAP deficiency in humans causes an X-linked lymphoproliferative syndrome. Nature 2006; 444: 110-114.

40. Beisner DR, Ch'en IL, Kolla RV, Hoffmann A, Hedrick SM. Cutting edge: innate immunity conferred by B cells is regulated by caspase-8. J Immunol 2005; 175: 3469-3473. 